\title{
Thermo-Responsive Chiral Column by Scissoring Motion from Rigid-Flexible Aromatic Rod Assembly
}

Shen Chen, ${ }^{b, c, \neq}$ Danfeng Deng, ${ }^{b, \neq}$ Siying Xie, ${ }^{a,}$ ShanShan $W u,{ }^{a,}$ Jing Peng, ${ }^{b,}$ Cong Ma, ${ }^{b}$ Ping $\mathrm{Xu},{ }^{b}$ Dongyan Tang, ${ }^{b}$ Xiaohong $\mathrm{Wu}^{,}{ }^{b}$ and Zhegang Huang ${ }^{a, *}$

a School of Chemistry and Chemical Engineering, Sun Yat-sen University, Guan gzhou 510275, P. R. China

${ }^{b}$ Department of Chemistry, Harbin Institute of Technology, Harbin 150001, P. R. China

${ }^{c}$ School of Science, Harbin University, Harbin 150086, P. R. China

‡ These authors (S. C., and D. D.) contributed equally to this work.

Author for correspondence

Prof. Zhegang Huang

School of Chemistry and Chemical Engineering, Sun Yat-sen University, Guangzhou 510275, P. R. China

E-mail: huangzhg3@mail.sysu.edu.cn 


\begin{abstract}
Here, we report thermo-responsive chiral columns from selfassembled coil-rod-coil molecules. The rigid rods self-assemble into multiplying dimers which stack on the top of one another to form non-chiral columnar crystal. Notably, the achiral crystal transforms into 2D hexagonally ordered liquid crystal with supramolecular chirality as heating. The reversible chiral switching behavior within columnar structures arises from scissoring motion from neighbored molecules driven by high thermal entropy of flexible chains relative to stiff-rod.
\end{abstract}

Keywords: $\pi$ stacking; self-assembly; chirality control; helical stacking; optical switch

The sensor of chirality based on self-aggregated helix has been of growing interest both for life and materials science due to their wide application potentials in interdisciplinary areas combining chemistry, biology and optical physics. $^{1-8}$ In the living system, it is known that DNA and proteins can produce mechanical signals through spontaneous motions in response to external stimuli. Like human DNA, generally, the artificial supramolecular nano-helix can be constructed by the assembly process of chiral molecules through hydrogen bonding, 9,10 तinteractions. ${ }^{13,14}$ Among them, chirality control of the helical architectures is one of the highlighted topics to mimic the molecular motions of complicated biological systems. The majority of these studies are reported in polymers, liquid crystalline phases, or host-guest complexes systems by changing the 
external conditions such as solvent, ${ }^{15,16}$ temperature, ${ }^{17,18}$ light, ${ }^{19-21} \mathrm{pH}^{22-25}$ or by interaction with specific guests. ${ }^{26-29}$ The senses of chirality in self-assembled systems can be ascribed to a change of helical twisting power (HTP) or a conformational change of the molecular backbones due to supramolecular aggregations. Previously, we have prepared a switchable helical fiber with reversible chiral-nonchiral states from the self-assembly of rigid flexible amphiphiles through intermolecular spring motion. ${ }^{30}$ We have also reported a tunable 2D organizaion of dumbbell-shaped amphiphiles based on different cross-sectional flexible segments by scissoring motion. ${ }^{31}$ The manipulation of 2D growth was attributed to the degree of twisted angle between two neighboring stacked rigid segments. Moreover, introducing chirality into flexible segment would produce a switchable column between crystal and liquid crystals resulting in the competition of microphase separation and $\pi-\pi$ interaction. Beyond the molecular design, herein, we report the formation of temperatureresponsive chiral columns self-assembled from the dumbbell-shaped coil-rodcoil triggered by molecular scissoring motion. For comparison, we investigated the derivative 2 based on strongly m stacked aromatic anthracene (scheme 1). 

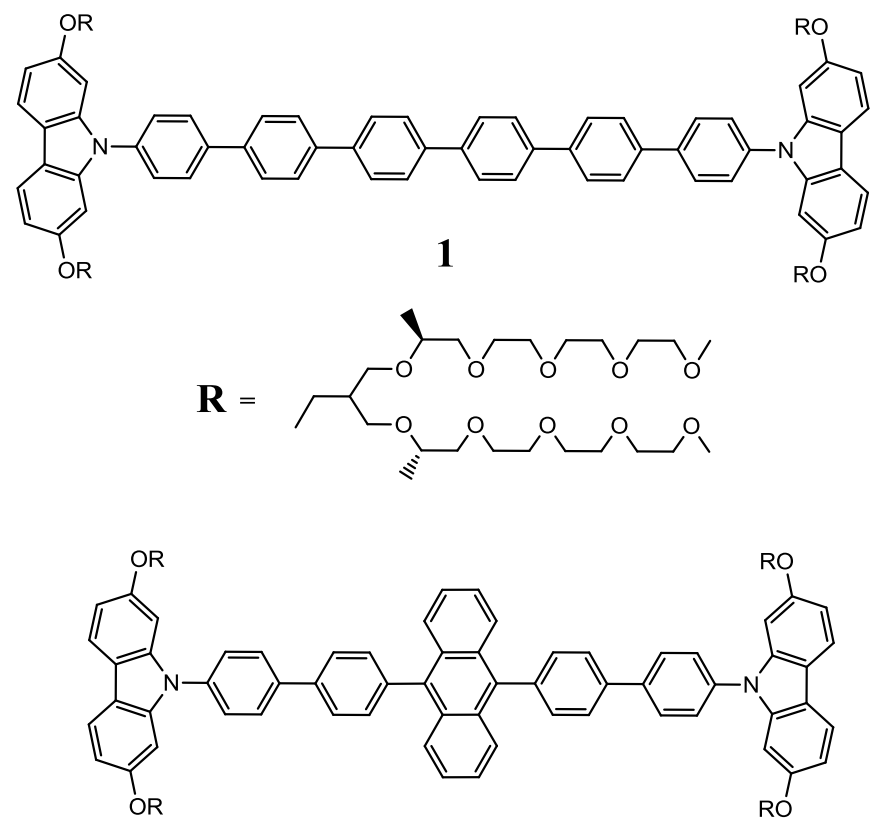

2

Scheme 1. Chemical structure of rigid-flexible amphiphiles 1 and 2.

The synthesis of $\mathbf{1}$ was performed stepwise according to previously reported methods, and meanwhile, $\mathbf{2}$ was synthesized from the preparation of antharacene bi-boronic acid and 9-arylcarbazole aromatic scaffold according to the procedure described previously. ${ }^{32}$ The final compound was successfully synthesized by Suzuki coupling with the adaptive boronic acid. These resulting molecules 1 and 2 were characterized by ${ }^{1} \mathrm{H}$ and ${ }^{13} \mathrm{C}$ NMR spectroscopy, and matrix-assisted laser desorption ionization time-of-flight (MALDI-TOF) mass spectroscopy and the data were shown to be in full agreement with the structures presented.

Molecule 1 which consists of dumbbell-shaped stiff rod block and flexible dendritic coil block can self-assemble into an aggregated structure due to microphase separation between each block on the solid film. Subsequently, the aggregation behavior of $\mathbf{1}$ in solid state was investigated by means of DSC, 
a

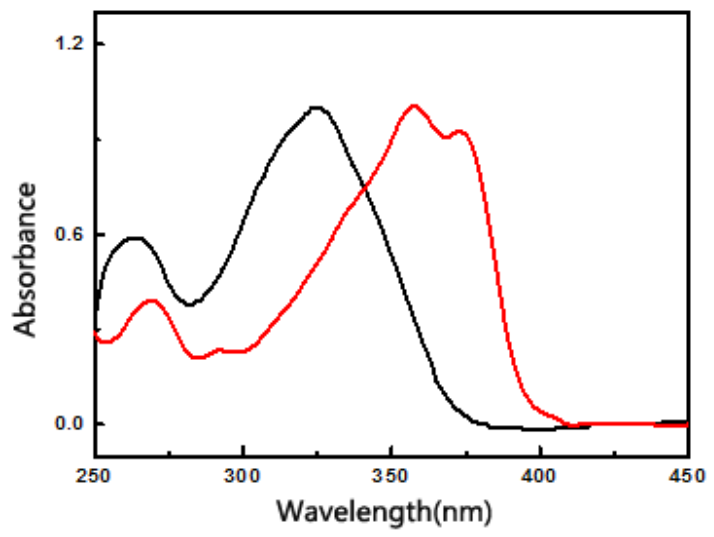

b

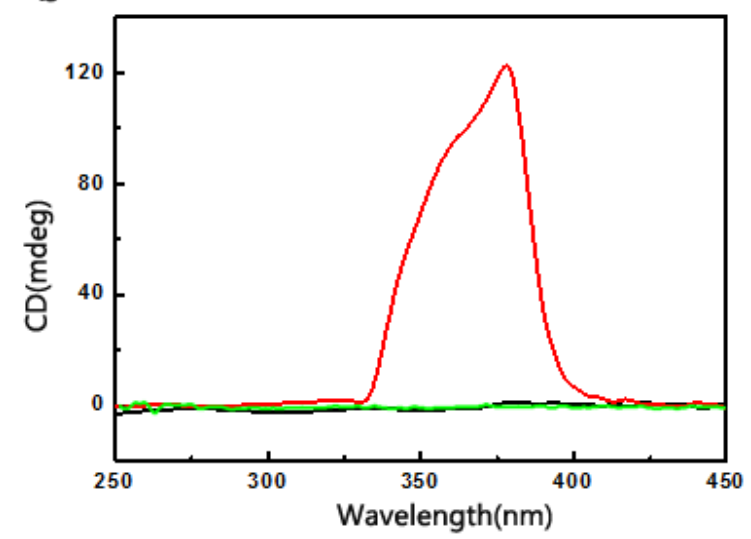

Fig. 1. a) The absorption spectra of 1 in $\mathrm{CHCl}_{3}(17 \mathrm{uM}$, black), and in solid film measured at $30{ }^{\circ} \mathrm{C}$ (red); b) $\mathrm{CD}$ spectra of 1 measured at $30{ }^{\circ} \mathrm{C}$ (black), at $70{ }^{\circ} \mathrm{C}$ (red), and 2 measured at 30 ${ }^{\circ} \mathrm{C}$ (green).

thermal optical polarized microscopy, UV-Vis spectroscopy, circular dichroism (CD), and X-ray scattering. Similar to the one described previously, $\mathbf{1}$ in chloroform (17 uM) shows an absorption maximum at $325 \mathrm{~nm}\left(\pi-\pi^{*}\right.$ transition) and two emissions at 397 and $410 \mathrm{~nm}$, suggesting the carbazole plane was twisted with hexa-p-phenylene at molecular dissolved state. ${ }^{30}$ In the solid film, the UV shows two maximum absorptions at 358 and $373 \mathrm{~nm}$ and fluorescence quenching (Fig. 1a and Fig. S1, Supplementary Information). The red shift can be ascribed to the existence of the effective conjugated length, indicating that the twisted aromatic rod transforms into co-planar conformations to induce strong aggregation in solid state. On slow cooling from the isotropic liquid to room temperature $(\mathrm{RT})$, a fernlike texture which corresponds to a typical columnar mesophase was observed (Fig. S2, Supplementary Information). Interestingly, when the column on a quartz plate was subjected to circular 


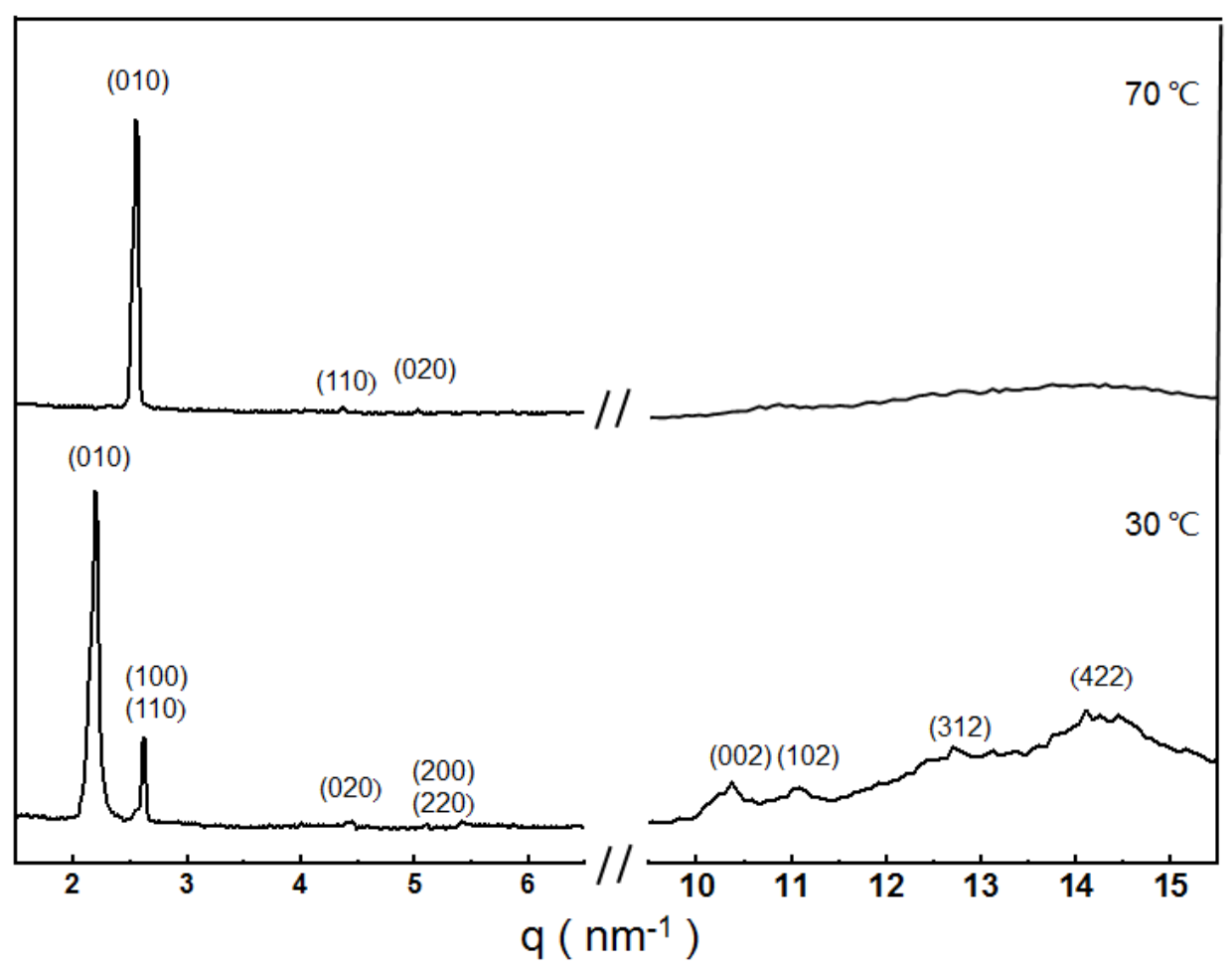

Fig. 2. X-ray diffraction patterns measured at different temperatures plotted against $q$ (= $4 \pi \sin 2 \theta / \lambda)$ in the oblique columnar structure at $30{ }^{\circ} \mathrm{C}$, and the hexagonal columnar structure at $70{ }^{\circ} \mathrm{C}$ for 1.

dichroism (CD) measurements at $30{ }^{\circ} \mathrm{C}$, no $\mathrm{CD}$ signals could be detected, indicating that the columnar objects are non-chiral even though 1 contains chiral side groups (Fig. 1b). To further understand the molecular packing within a column, we performed X-ray scattering experiments. The small-angle X-ray diffraction (SAXS) pattern of $\mathbf{1}$ displayed a number of sharp reflections at $30^{\circ} \mathrm{C}$ with some peaks at wide angle, an indication of the existence of a highly ordered nanoscopic structure with three distinct lattice parameters (Fig. 2). These reflections indeed can be indexed to an oblique columnar crystalline structure with a characteristic angle of $\gamma=65^{\circ}$ and lattice parameters $a=2.7 \mathrm{~nm}$, $b=3.2 \mathrm{~nm}, c=1.2 \mathrm{~nm}$ (Table S1, Supplementary Information). In order to better 
understand the packing structure, we calculated the number $(n)$ of molecules in one column. From the measured density $\left(1.17 \mathrm{~g} \cdot \mathrm{cm}^{-3}\right)$ and the lattice volume (Table 1), it is estimated that approximately two molecules were filled in a slice of each column. Based on the extended aromatic length (3.5 nm by CoreyPauling-Koltun (CPK) molecular model) and 002, 102, 312, 422 such as three dimensional peaks which observed in wide angle (Fig. 2), we consider that the neighbored two molecules are stacked along $\mathrm{c}$ axis with alternative arrangement through strong $\pi-\pi$ stacking to form crystalline ordering, thus frustrating the chiral transfer from the side groups to the aromatic cores, leading to a non-chiral column.

Table 1. Thermal transtions of dumbbell-shaped molecules (data are from heating and cooling scans) ${ }^{[a]}$

\begin{tabular}{|c|c|c|c|c|}
\hline \multirow[b]{2}{*}{ Molecules } & \multirow[b]{2}{*}{$\rho^{[\mathrm{b}]}\left[\mathrm{g} / \mathrm{cm}^{3}\right]$} & \multirow[b]{2}{*}{$\mathrm{F}_{\mathrm{rod}}^{[c]}$} & \multicolumn{2}{|c|}{ Phase transition $\left[{ }^{\circ} \mathrm{C}\right]$ and corresponding enthalpy changes $[\mathrm{kJ} / \mathrm{mol}]$} \\
\hline & & & Heating & Cooling \\
\hline 1 & 1.17 & 0.27 & $\operatorname{Cr} 63.6(5.1) \quad H c 111.1(9.3) \mathrm{i}$ & I $100.8(-8.9) \quad \mathrm{Hc} 46.2(-5.2) \mathrm{Cr}$ \\
\hline 2 & 1.14 & 0.28 & $\operatorname{Cr} 123.5(6.8) \mathrm{i}$ & I $109.4(-8.2) \mathrm{Cr}$ \\
\hline
\end{tabular}

[a] Data are from heating and cooling scans. Cr: alternartively stacked crystal;Hc: helical columnar liquid crystal. [b] $\rho=$ molecular density. $[\mathrm{c}] f_{\text {rod }}=$ rod volume fraction.

The formation of multiplying rod packing with the flexible dendritic exterior suggests that the columnar crystals would exhibit thermo-responsive behavior because the higher entropy of flexible chains relative to the stiff-rod segments requires a larger interfacial area to reduce enhanced space crowding in the flexible domains. Indeed, the DSC thermograms showed that crystal 1 melts 
into anisotropic liquid crystals at $64{ }^{\circ} \mathrm{C}$ followed by isotropic liquid (Fig. S3, Supplementary Information). In contrast to low temperature, the CD at liquid crystalline phase gives positive cotton effect in the spectral range of the aromatic units (Fig. 1), indicative of the formation of helical stacks of the rod segments with a preferred handedness ${ }^{33}$. To understand the $2 \mathrm{D}$ structures, we performed X-ray scattering experiment at high temperature. As heating, the several reflections of $\mathbf{1}$ in the wide-angle gradually defused with some new peaks at small angle, suggesting a liquid crystal was formed with reduced $\pi-\pi$ interaction between neighbored rods. However, it is notable that both of the Xray scattering patterns, regardless of temperature, showed a reflection at the same position about $10.7 \mathrm{~nm}^{-1}$ (Fig. 2 and Fig. S4, Supplementary Information), indicating the $\pi$ stacking distance of neighboring rods is nearly unchanged. Upon heating to $70{ }^{\circ} \mathrm{C}$, both 100 and 010 peaks merged into a new reflection accompanied by two peaks at middle-angle and these three diffractions at high temperature could be indexed as a $2 \mathrm{D}$ hexagonal columnar structure with a lattice constant of $2.9 \mathrm{~nm}$ (Table S2, Supplementary Information). Compared with the oblique columnar, the hexagonal ordering has an increased parameter ratio $(a / b)$, whereas a decreased anisotropic ability, suggesting the reduced $\pi$ interaction take place in column plane.

Based on these results, the unique feature of chiral induction can be explained by the competition between $\pi-\pi$ stacking and microphase separation. The rod segments within columnar structure at lower temperature have a strong tendency of anisotropic crystalline ordering to drive the rod segments to aggregate in one dimension with $A B A B$ arrangement through microphase 
separation between incompatible components and $\pi-\pi$ stacking interaction, thus, frustrating the chiral transfer from the side groups to the aromatic cores, leading to a non-chiral column. As heating, however, higher thermal motion of flexible chains relative to the stiff-rod block requires a larger interfacial area. To reduce this enhanced crowding at interphase, the alternatively stacked rod separated more to reduce anisotropic interaction leading to neighbored rod rotated one dimension to minimize a steric repulsion between bulky dendritic segments.

To gain insight into the role of the $\pi-\pi$ stacking interactions between neighbored aromatic segments in the switchable behavior of chirality, compound 2 based on an antharacene aromatic unit was prepared with the aim of frustration of chiral amplification by strong anisotropic interaction. The dumbbell-shaped molecule $\mathbf{2}$ was also observed form columnar crystals that melt into isotropic liquid upon $87^{\circ} \mathrm{C}$. As expected, the solid film based on selfassembly of $\mathbf{2}$ did not show any CD signal in columnar crystalline phase (Fig. 1b). To obtain more structure information of the solid phase, the WAXD analysis was performed at RT. Both in small- and wide-angle a number of diffractions were observed. The summary of the diffraction peaks and their indexation can be found in the supporting information. In the small-angle, 2 shows two kinds of equidistant reflections that can be indexed based on a 2D oblique lattice: $a=$ $2.4 \mathrm{~nm}, b=3.8 \mathrm{~nm}, \quad \gamma=71^{\circ}$ (Fig. S5, Supplementary Information). The large number of ( $h k l)$ diffractions at wide-angle, particularly those with $/ \neq 0$, suggests the existence of crystalline nature of the columns. These (hkl) with (001) 
diffractions confirm that there is repective periodicity with $0.82 \mathrm{~nm}$ along $c^{*}$ axis. According to the cell parameter, two molecules were calculated within one unit cell of the columnar crystals. From the molecular parameters, it is apparent that the molecular core covers the $a, b$ plane allowing neighboured molecules along $c^{*}$ axis with strong anisotropic interaction to produce periodic columnar crystals (Fig. S6, Supplementary Information). The comparison between $\mathbf{1}$ and $\mathbf{2}$, strongly supports the appropriate competition based on $\pi$ stacking and microphase separation plays a crucial role in the reversible chiral switching in columnar aggregates.

\section{Top view}

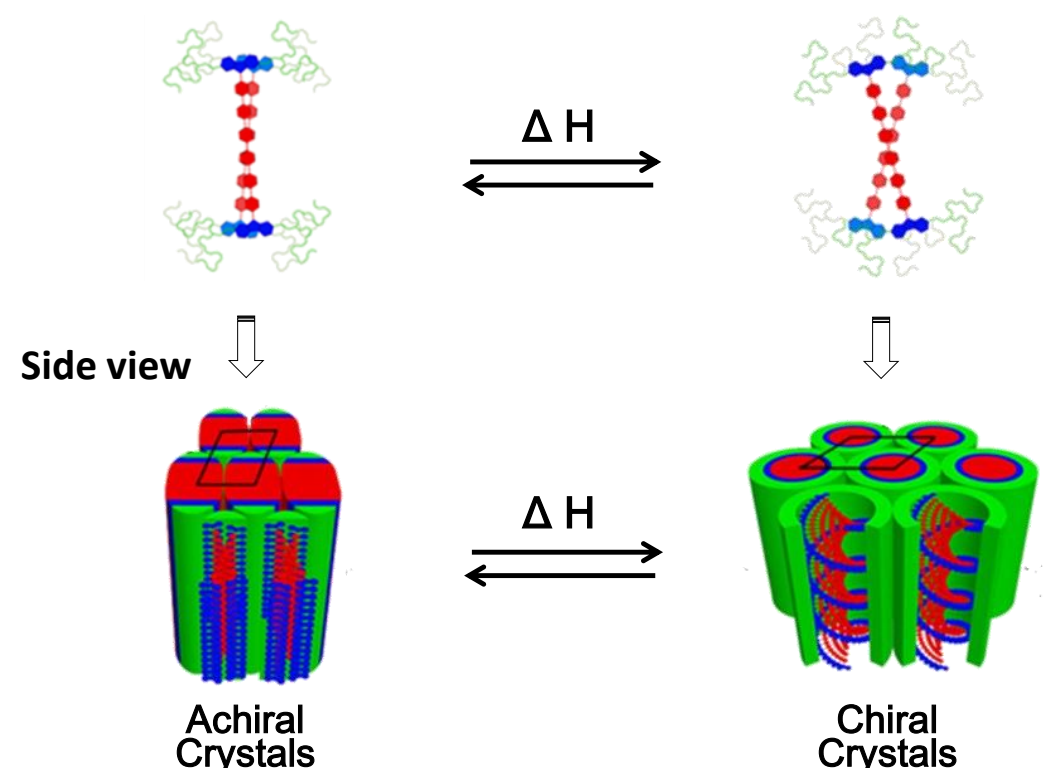

Fig. 3. Schematic representation of thermo-responsive organization of $\mathbf{1 .}$

In summary, we have demonstrated herein that the coil-rod-coil molecules can self-assembles into multi-twisted ordering. At room temperature, the neighboring molecules show strong $\pi$ interaction to self-assemble into alternatively stacked columnar crystals. The multiplying crystals were shown to 
be temperature-responsive to convert into helical columnar liquid crystals by scissoring motion of neighbored molecules (Fig. 3). It is notable that the

switching behavior of chirality within columnar structure by dynamic molecular motion represents a remarkable contrast to other helical columnar structures that dissolve into molecular components. Such optical switch by mechanical motion on thin film may provide a new strategy for the construction of dynamic nano devices or optical motor with desired functions.

\section{Acknowledgements}

This work was supported by the NSF (21302030), Key Laboratory of Functional Inorganic Material Chemistry (Heilongjiang University), Ministry of Education (2013kf05), Open Project of State Key Laboratory of Supramolecular Structure and Materials (sklssm2015011). Z.H. acknowledges the financial support from Sun Yat-sen University.

\section{References}

[1] Busseron E, Ruff Y, Moulin E, Giuseppone N. Supramolecular self-assemblies as functional nanomaterials. Nanoscale 2013;5:7098-7140. DOI: 10.1039/c3nr02176a.

[2] Wang Q, Zhang X, Zheng J, Liu D. Self-assembled peptide nanotubes as potential nanocarriers for drug delivery. RSC Adv 2014;4:25461-25469. DOI: 10.1039/c4ra03304c.

[3] Hernandez-Garcia A, Kraft DJ, Janssen AFJ, Bomans PHH, Sommerdijk NAJM, Thies-Weesie DME, et al. Design and self-assembly of simple coat proteins for artificial viruses. Nat Nanotechnol 2014;9:698-702. DOI: 10.1038/NNANO.2014.169. 
[4] Faul CFJ. Ionic self-assembly for functional hierarchical nanostructured materials. Acc Chem Res 2014;47:3428-3438. DOI: 10.1021/ar500162a.

[5] Roy B, Bairi P, Nandi AK. Supramolecular assembly of melamine and its derivatives: nanostructures to functional materials. RSC Adv 2014;4:1708-1734. DOI: 10.1039/c3ra44524k.

[6] Kang S, Huynh T, Xia Z, Zhang Y, Fang H, Wei G, Zhou R. Hydrophobic interaction drives surface-assisted epitaxial assembly of amyloid-like peptides. J Am Chem Soc 2013;135:3150-3157. DOI: 10.1021/ja310989u.

[7] Tejedor RM, Serrano J, Oriol L. Photocontrol of supramolecular architecture in azopolymers: Achiral and chiral aggregation. Eur Polym J 2009;45:2564-2571. DOI: 10.1016/j.eurpolymj.2009.05.010.

[8] Miyake H, Tsukube H. Coordination chemistry strategies for dynamic helicates: time-programmable chirality switching with labile and inert metal helicates. Chem Soc Rev 2012;41:6977-6991. DOI: 10.1039/c2cs35192g.

[9] García F, Buendía J, Sánchez L. Supramolecular ribbons from amphiphilic trisamides self-Assembly. J Org Chem 2011;76:6271-6276. DOI: 10.1021/jo201055t.

[10] Lu X, Guo Z, Sun C, Tian H, Zhu W. Helical assembly induced by hydrogen bonding from chiral carboxylic acids based on perylene bisimides. J Phys Chem B 2011;115:10871-10876. DOI: 10.1021/jp2064968.

[11] Tominaga M, Azumaya I. Fibrous architectures and organogels of tris(phenylethynylphenyl)adamantane molecules with amino acid moieties: their solvato-controlled helicity induction. Chirality 2011;23:E59-E64. DOI: 10.1002/chir.20964

[12] Virkki MT, Peters C, Nilsson D, Sörensen T, Cristobal S, Wallner B, Elofsson A. The positive inside rule is stronger when followed by a transmembrane helix. $J$ Mol Biol 2014;426:2982-2991. DOI: 10.1016/j.jmb.2014.06.002.

[13] Kohlstedt KL, Solis F, Vernizzi G, Olvera DLCM. Spontaneous Chirality via long-range electrostatic forces. Phys Rev Lett 2007;99:030602. DOI : 10.1103/PhysRevLett.99.030602.

[14] Xiao J, Xu J, Cui S, Liu H, Wang S, Li Y. Supramolecular helix of an amphiphilic pyrene derivative induced by chiral tryptophan through electrostatic interactions. Org Lett 2008;10:645-648. DOI: 10.1021/ol7030087.

[15] Furusho Y, Yashima E. Synthesis and function of double-stranded helical polymers and oligomers. Macromol Rapid Commun 2011;32:136-146. DOI: 10.1002/marc.201000533. 
[16] Guo Q, Wang J, Zhu L, Wei Z. Modulating the helicity of sugar-substituted perylene diimide self-assemblies by solvent polarilities. Chin J Chem 2015;33:95-100. DOI: 10.1002/cjoc.201400218.

[17] Huang Z, Kang SK, Banno M, Yamaguchi T, Lee D, Seok C, Yashima E, Lee M. Pulsating tubules from noncovalent macrocycles. Science 2012;337:1521-1526. DOI: 10.1126/science.1224741.

[18] Kumar J, Nakashima T, Kawai T. Inversion of supramolecular chirality in bichromophoric perylene bisimides: influence of temperature and ultrasound. Langmuir 2014;30:6030-6037. DOI: 10.1021/la500497g.

[19] Kim J, Lee J, Kim WY, Kim H, Lee S, Lee HC, Lee YS, Seo M, Kim SY. Induction and control of supramolecular chirality by light in self-assembled helical nanostructures. Nat Commun 2015;6:6959. DOI: 10.1038/ncomms7959.

[20] Nakashima T, Yamamoto K, Kimura Y, Kawai T. Chiral photoresponsive tetrathiazoles that provide snapshots of folding states. Chem Eur J 2013;19:16972-16980. DOI: 10.1002/chem.201302564.

[21] Wang Y, Zhou D, Li H, Li R, Zhong Y, Sun X, Sun X. Hydrogen-bonded supercoil self-assembly from achiral molecular components with light-driven supramolecular chirality. J Mater Chem C 2014;2:6402-6409. DOI: 10.1039/c4tc00649f.

[22] Kurouski D, Lombardi RA, Dukor RK, Lednev IK, Nafie LA. Direct observation and pH control of reversed supramolecular chirality in insulin fibrils by vibrational circular dichroismw. Chem Commun 2010;46:7154-7156. DOI: $10.1039 / \mathrm{c0cc02423f.}$

[23] Duan P, Qin L, Zhu X, Liu M. Hierarchical self-assembly of amphiphilic peptide dendrons: evolution of diverse chiral nanostructures through hydrogel formation over a wide pH range. Chem Eur J 2011;17:6389-6395. DOI: 10.1002/chem.201003049.

[24] Kurouski D, Lu X, Popova L, Wan W, Shanmugasundaram M, Stubbs G, Dukor RK, Lednev IK, Nafie LA. Is supramolecular filament chirality the underlying cause of major morphology differences in amyloid fibrils? $J \mathrm{Am}$ Chem Soc 2014;136:2302-2312. DOI : 10.1021/ja407583r.

[25] Zhan X, Tamhane K, Bera T, Fang J. Transcription of pH-sensitive supramolecular assemblies into silica: from straight, coiled, and helical tubes to single and double fan-like bundles. J Mater Chem 2011;21:1397313977. DOI: 10.1039/c1jm10937e.

[26] Wang Y, Huang Z, Kim Y, He Y, Lee M. Guest-driven inflation of self-assembled nanofibers through hollow channel formation. J Am Chem Soc 2014;136:16152-16155. DOI: 10.1021/ja510182x. 
[27] Ryu J, Kim H, Huang Z, Lee E, Lee M. Self-assembling molecular dumbbells: from nanohelices to nanocapsules triggered by guest intercalation. Angew Chem Int Ed 2006;45:5304-5307. DOI: 10.1002/anie.200600971.

[28] Tazawa T, Yagai S, Kikkawa Y, Karatsu T, Kitamura A, Ajayaghosh A. A complementary guest induced morphology transition in a two-component multiple H-bonding self-assembly. Chem Commun 2010;46:10761078. DOI: 10.1039/b921959e.

[29] Meudtner RM, Hecht S. Helicity inversion in responsive foldamers induced by achiral halide ion guests. Angew Chem Int Ed 2008;47:4926-4930. DOI: 10.1002/anie.200800796.

[30] Huang Z, Kang S, Lee M. Induction of supramolecular chirality in self-assembled nanofibers triggered by environmental change. J Mater Chem 2011;21:15327-15331. DOI: 10.1039/c1jm12683k.

[31] Huang Z, Ryu J, Lee E, Lee M. Tunable columnar organization by twisted stacking of end-capped aromatic rods. Chem Mater 2007;19:6569-6574. DOI: 10.1021/cm7028189.

[32] Chen S, Ma C, Huang Z, Lee M. Controlled helicity of the rigid-flexible molecular assembly triggered by water addition: from nanocrystal to liquid crystal gel and aqueous nanofibers. $J$ Phys Chem C 2014;118:81818186. DOI: $10.1021 / \mathrm{jp} 500366 \mathrm{w}$.

[33] Fujiki, M. Helix magic. Thermo-driven chiroptical switching and screw-sense inversion of flexible rod helical polysilylenes. J Am Chem Soc 2000;122:3336-3343. DOI: 10.1021/ja9938581. 


\section{Graphical abstract}

\section{Thermo-Responsive Chiral Column by Scissoring Motion from Rigid-Flexible Aromatic Rod}

\section{Assembly}

Shen Chen, ${ }^{b, c, \neq}$ Danfeng Deng, ${ }^{b, \neq}$ Siying Xie, ${ }^{a,}$ ShanShan Wu, ${ }^{a,}$ Jing Peng, ${ }^{b,}$ Cong Ma, ${ }^{b}$ Ping $\mathrm{Xu},{ }^{b}$ Dongyan Tang, ${ }^{b}$ Xiaohong $\mathrm{Wu},{ }^{b}$ and Zhegang Huang ${ }^{a, *}$

Text for the table of contents
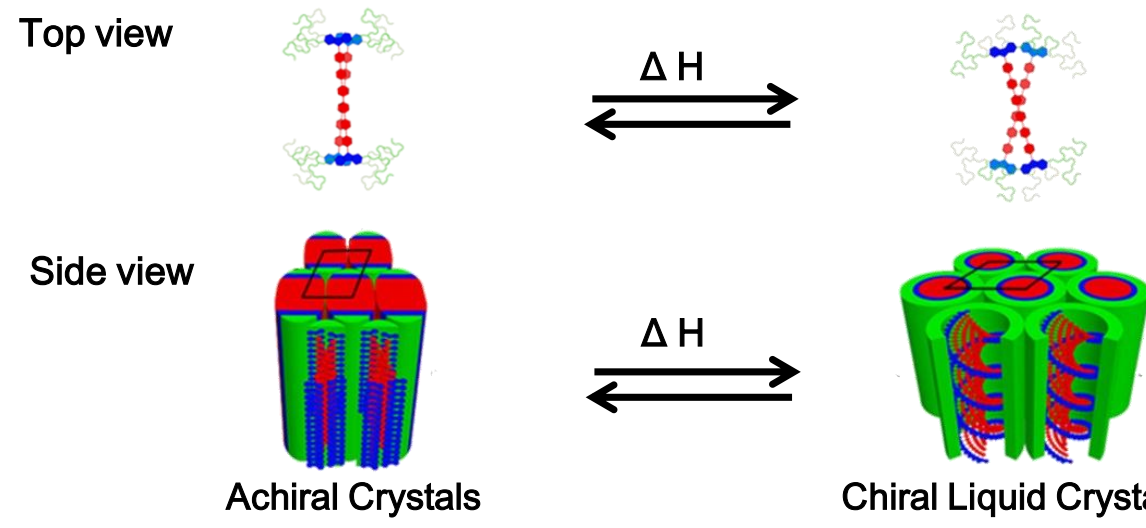

Chiral Liquid Crystals 